\title{
Low-grade appendiceal mucinous neoplasms observed by magnifying endoscopy
}

Appendiceal mucinous neoplasms are the second most common tumors after carcinoid tumors in all excised appendices [1]. Low-grade appendiceal mucinous neoplasms are often found incidentally $(\sim 50 \%)$, first discovered on radiography, endoscopy, or during surgery [2]. On endoscopy, they often appear as submucosal tumor-like elevations at the appendicular orifice [3]; there are no reports of associated epithelial changes. Herein we report two cases of endoscopically observed epithelial changes in low-grade appendiceal mucinous neoplasms ( $\triangleright$ Video 1 ).

Case 1: A 72-year-old woman underwent colonoscopy for contrast accumulation in the appendix on ${ }^{18} \mathrm{~F}$-fluorodeoxyglucose positron emission tomographycomputed tomography ( $\triangleright$ Fig. $\mathbf{1} \mathbf{a}, \mathbf{b}, \mathbf{c}$ ). Colonoscopy revealed a slightly elevated whitish lesion covered with a mucus cap in the cecum at the appendiceal orifice ( $\bullet$ Fig.2a,b). Magnifying narrow-band imaging (NBI) showed no vessel pattern and regular, wavy, elongated surface structures ( $\triangleright$ Fig. 2 c). Using chromoendoscopy with indigo carmine, the boundary of the lesion was clearly visualized ( Fig. 2d). Magnifying red dichromatic imaging with indigo carmine clearly showed regular, wavy, elongated, branched surface structures ( $>$ Fig. 2 e). Magnifying chromoendoscopy using crystal violet showed a wavy, branched pit, although the staining was not as clear as with other methods, probably owing to adherent mucus ( $\triangleright$ Fig. 2f). Histopathological examination following ileocecal resection revealed a low-grade appendiceal mucinous neoplasm ( $\mathbf{F i g . 3 a , b ) . ~}$

Case 2: A 74-year-old man underwent colonoscopy for appendiceal enlargement on computed tomography that showed a similar lesion as described in case 1 ( Fig. 4 a). Magnifying NBI, chromoendoscopy with indigo carmine, and magnifying chromoendoscopy using crystal violet showed the same findings as in case 1 ( Fig. $\mathbf{4 b}$ b, c, d). Following

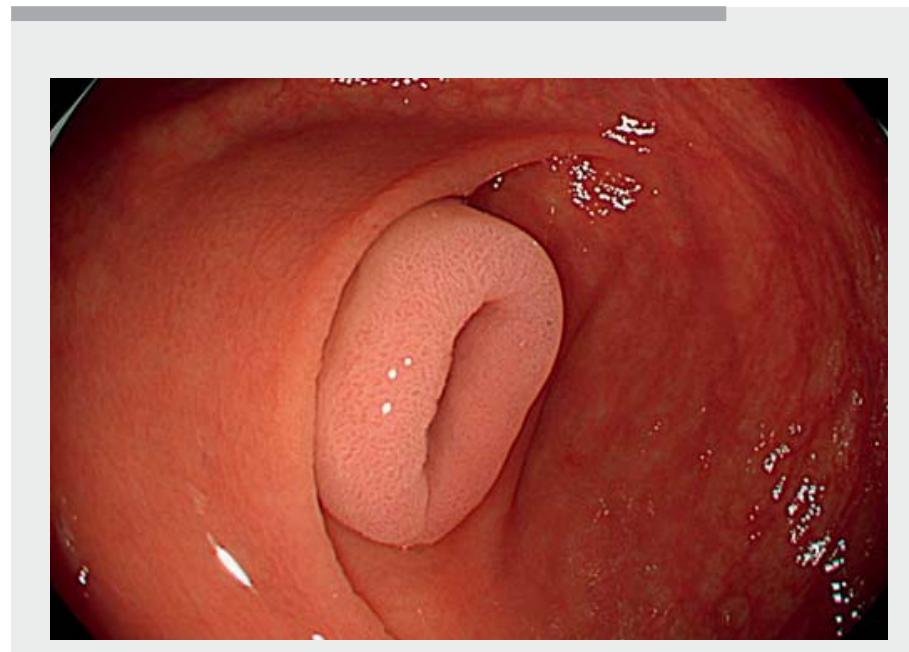

Video 1 Low-grade appendiceal mucinous neoplasms observed on magnifying endoscopy.

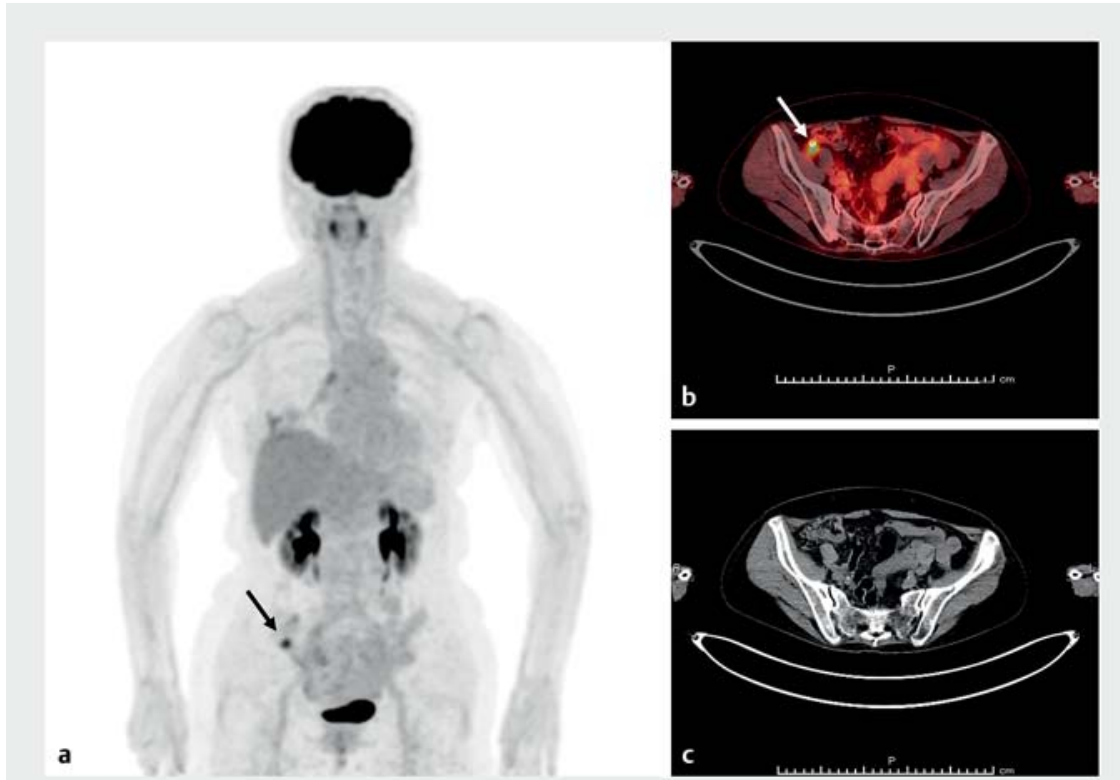

Fig. 1 a, b ${ }^{18}$ F-fluorodeoxyglucose positron emission tomography-computed tomography showing accumulation of the radiotracer in the appendix (arrow). c Computed tomography showing no lesions.

ileocecal resection, histopathology revealed a low-grade appendiceal mucinous neoplasm ( $\triangleright$ Fig.5a,b) with some adenocarcinoma components in the tail of the appendix.
In these cases, low-grade appendiceal mucinous neoplasms were observed as whitish, slightly elevated lesions covered with a mucus cap, and no blood vessels could be identified. The findings were 

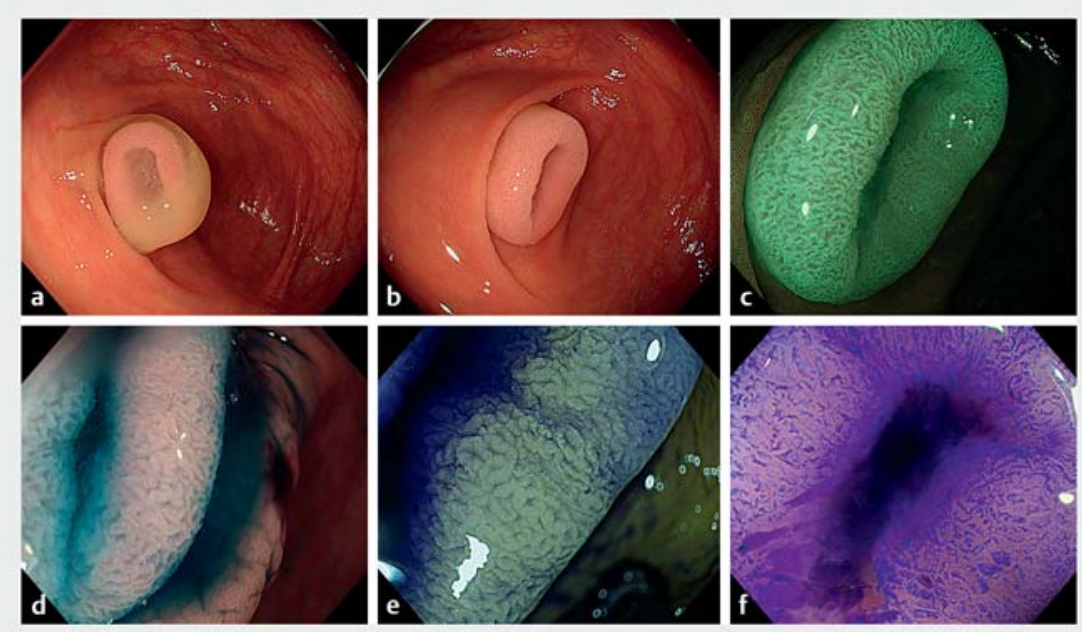

- Fig. 2 Endoscopic images showing a slightly elevated whitish lesion covered with a mucus cap in the cecum near the appendiceal orifice. a, b White light. c Magnifying narrow-band imaging. d Magnifying chromoendoscopy using indigo carmine. e Magnifying red dichromatic imaging with indigo carmine. fMagnifying chromoendoscopy using crystal violet staining.

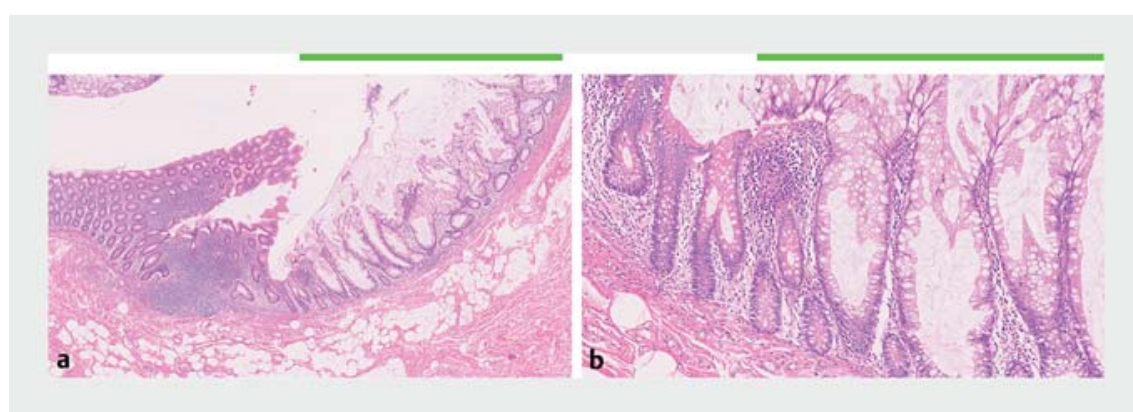

- Fig. 3 Histological examination (hematoxylin and eosin stained). a The distribution of lesions in the resection specimen is shown along with the boundary between normal mucosa and low-grade appendiceal mucinous neoplasm (the green line shows the area of low-grade appendiceal mucinous neoplasm with prominent mucous adhesion). $\mathbf{b}$ A magnified view of the area of low-grade appendiceal mucinous neoplasm; the mucinous epithelial cells are filiform with low-grade cytological atypia. more similar to serrated lesions than adenomas.

Endoscopy_UCTN_Code_CCL_1AD_2AB

Competing interests

The authors declare that they have no conflict of interest.

The authors

Kengo Kasuga, Keigo Sato, Yu Hashimoto, Hirohito Tanaka, Hiroko Hosaka $\odot$, Shiko Kuribayashi, Toshio Uraoka ${ }^{\circledR} \odot$

Department of Gastroenterology and Hepatology, Gunma University Graduate School of Medicine

\section{Corresponding author}

\section{Toshio Uraoka, MD}

Department of Gastroenterology and Hepatology, Gunma University Graduate School of Medicine, 3-39-22 Showa-machi, Maebashi 371-8514, Japan

Fax: +81-27-220-8137

uraoka@gunma-u.ac.jp 

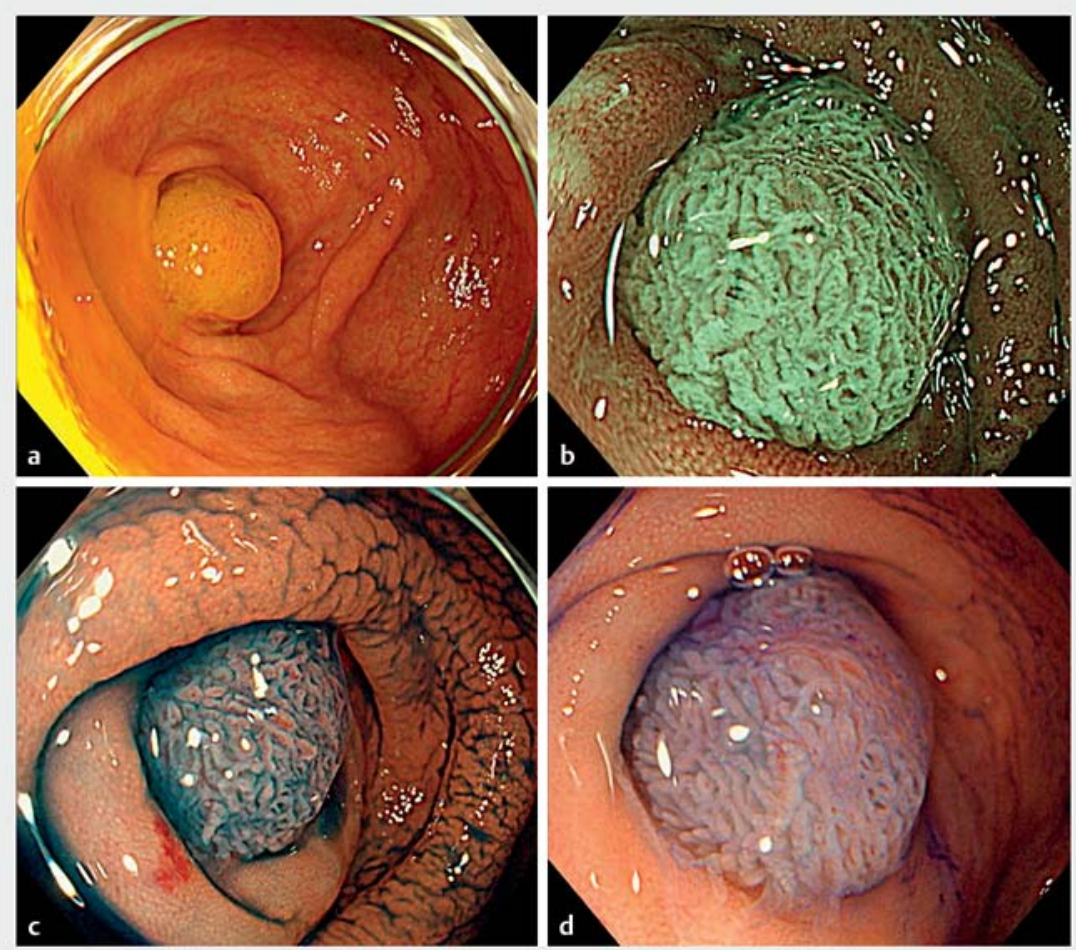

- Fig. 4 Endoscopic images showing a slightly elevated whitish lesion covered with a mucus cap in the cecum near the appendiceal orifice. a White light. b Magnifying narrow-band imaging. c Magnifying chromoendoscopy using indigo carmine. d Magnifying chromoendoscopy using crystal violet staining.
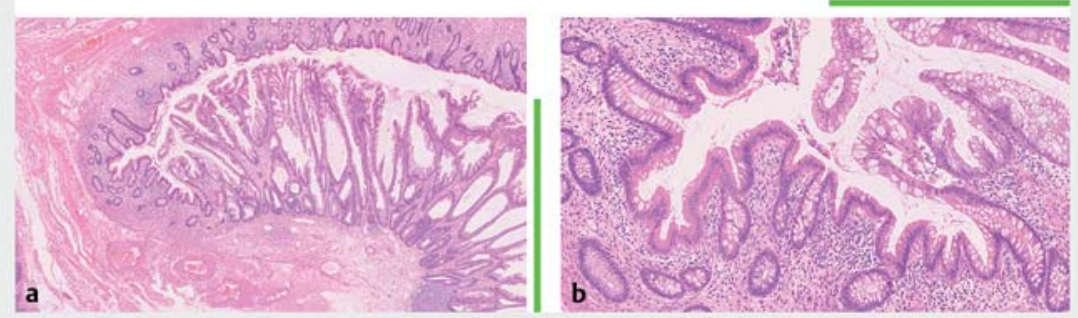

Fig. 5 Histological examination (hematoxylin and eosin stained). a The distribution of lesions in the resection specimen is shown along with the boundary between the normal mucosa and low-grade appendiceal mucinous neoplasm (the green line shows the area of low-grade appendiceal mucinous neoplasm). b A magnified view of the area of low-grade appendiceal mucinous neoplasm; the mucinous epithelial cells are villous with low-grade cytological atypia.

\section{References}

[1] Connor S], Hanna GB, Frizelle FA. Appendiceal tumors: retrospective clinicopathologic analysis of appendiceal tumors from 7,970 appendectomies. Dis Colon Rectum 1998; 41: $75-80$

[2] Rojnoveanu G, Ghidirim G, Mishin I et al. Preoperatively diagnosed mucocele of the appendix. Chirurgia (Bucur) 2014; 109: 416-420

[3] Kapila A. An incidental discovery of lowgrade appendiceal mucinous neoplasm. Perm J 2014; 18: e153-3154. doi:10.7812/ tpp/14-077

\section{Bibliography}

Endoscopy 2022; 54: E704-E706

DOI 10.1055/a-1769-4850

ISSN 0013-726X

published online 28.2.2022

(C) 2022. Thieme. All rights reserved.

Georg Thieme Verlag KG, Rüdigerstraße 14, 70469 Stuttgart, Germany

\section{ENDOSCOPY E-VIDEOS}

https://eref.thieme.de/e-videos

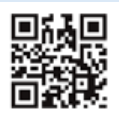

Endoscopy E-Videos is an open access online section, reporting on interesting cases and new techniques in gastroenterological endoscopy. All papers include a high quality video and all contributions are freely accessible online. Processing charges apply (currently EUR 375), discounts and wavers acc. to HINARI are available.

This section has its own submission website at

https://mc.manuscriptcentral.com/e-videos 